\title{
Hidden in plain sight: MSM engaging in Chemsex as an emerging risk group for STI/HIV
}

Evers, YJ1,2, Van Liere, GAFS ${ }^{1,2}$, Hoebe, CJPA 1,2 , Dukers-Muijrers, NHTM 1,2
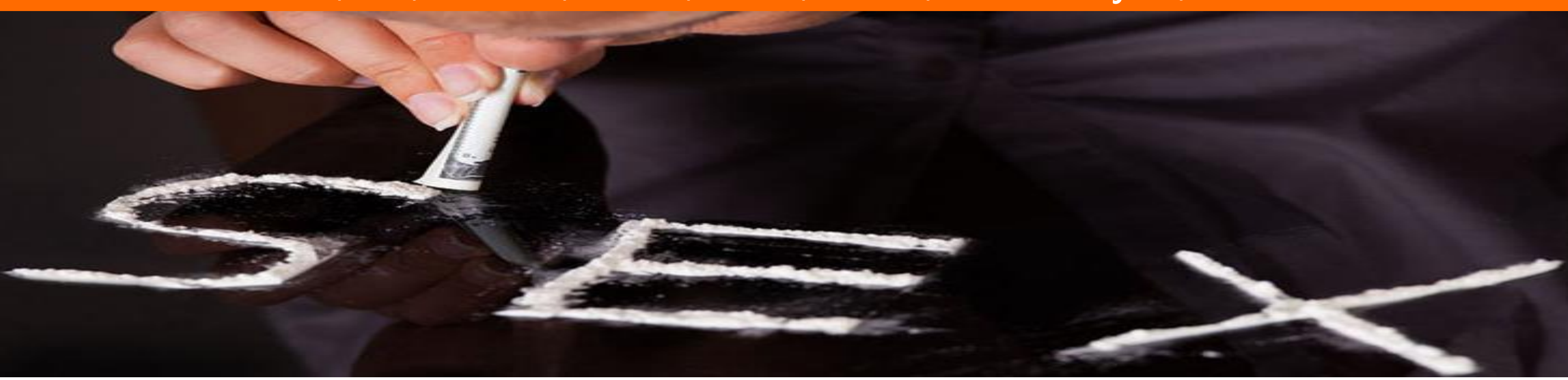

\section{Background}

- Men who have sex with men (MSM) are increasingly using drugs during sex ('Chemsex'). Chemsex might contribute to the high incidence of STI/HIV among MSM in many developed countries.

- Most previous research focused on MSM in the UK and four typical chemsex drugs: crystal meth, mephedrone, GHB and GBL.

- The current study uniquely examines a wide variety of drug types used during sex, and their associations with STI/HIV, using STI/HIV laboratory test results of MSM visiting two Dutch STI clinics.

\section{Methods}

\section{Study population:}

\begin{tabular}{|c|}
$\begin{array}{c}348 \text { MSM recruited } \\
\text { at two STI clinics }\end{array}$ \\
\hline $\begin{array}{c}250 \mathrm{MSM}(71.8 \%) \text { completed } \\
\text { the online questionnaire }\end{array}$ \\
\hline
\end{tabular}

- Participants were representative for MSM STI population regarding age and STI test results (data not shown).

- Median age participants: 35 years

\section{Analysis:}

- Questionnaire data and STI client registry data, including STI/HIV test results.

- Outcome: any STI (CT, NG, syphilis, hepatitis B, or HIV at most recent consultation of clients).

- Determinants: chemsex (any drug use during sex, excluding alcohol, cannabis, erection medicines), drug types used during sex in preceding six months.

- Univariate logistic regression analyses.

\section{Results}

- Prevalence any STI: $19.2 \%$

- Prevalence chemsex: $51.6 \%(121 / 250)$

- Top six most used drugs during sex:
1. Poppers:
$42.0 \%(105 / 250)$
2. XTC/MDMA:
$27.2 \%(68 / 250)$
3. GHB/GBL:
$25.6 \%(64 / 250)$
4. Ketamine:
$12.4 \%(31 / 250)$
5. Speed:
$10.8 \%(27 / 250)$
6. Cocaine:
$9.2 \% \quad(23 / 250)$

- Crystal meth (2.0\%; 5/250), mephedrone (1.6\%; $4 / 250)$, and research chemicals $(3.2 \% ; 8 / 250)$ rarely used during sex.

\begin{tabular}{|lll|}
\hline $\begin{array}{l}\text { Drug use during sex } \\
\leq 6 \text { months }\end{array}$ & Any STI & Univariate OR \\
\hline Chemsex & $24.8 \%(32)$ & $2.17(1.12-4.19)^{*}$ \\
\hline Four chemsex drugs & $36.9 \%(24)$ & $3.93(2.03-7.61)^{* * *}$ \\
\hline Poppers & $26.7 \%(28)$ & $2.27(1.20-4.31)^{* *}$ \\
\hline XTC/MDMA & $35.3 \%(24)$ & $3.59(1.86-6.93)^{* * *}$ \\
\hline GHB/GBL & $35.9 \%(23)$ & $3.61(1.86-7.00)^{* * *}$ \\
\hline Ketamine & $35.5 \%(11)$ & $2.71(1.20-6.12)^{*}$ \\
\hline Speed & $25.9 \%(7)$ & $1.55(0.62-3.92)^{* * *}$ \\
\hline Cocaine & $47.8 \%(11)$ & $4.71(1.93-11.47)^{* *}$ \\
\hline & & $*<0.05,{ }^{* *}<0.01,{ }^{* * *}<0.001$ \\
\hline
\end{tabular}

\section{Conclusion}

- Over half of MSM visiting Dutch STI clinics recently engaged in chemsex and this was associated with any STI, indicating the need for monitoring and improved care and prevention.

- A broader definition of chemsex, incorporating more drug types than the four typical chemsex drugs, might be more appropriate to reflect the STI risks. 